\title{
Designing AI-assisted interactive map displays to explore spatial information
}

\author{
Merve Keskin $^{\text {a, }}$, Pyry Kettunen ${ }^{\text {a }}$ \\ ${ }^{a}$ Department of Geoinformatics and Cartography, Finnish Geospatial Research Institute (FGI, NLS), merve.keskin@nls.fi, \\ pyry.kettunen@nls.fi \\ * Corresponding author
}

Keywords: interactive map displays, artificial intelligence, geoexploration, eye tracking, usability

\begin{abstract}
:
Although nowadays we are able to produce large volumes of spatial or geo-referenced data through fast data collection methods (e.g. mobile phones, sensors, GPS), making sense of the collected big spatial data still remains a big challenge to people. Visual analytics and exploratory geovisualization tools have been employed to identify the unknown geospatial phenomena within unstructured and inconsistent data and serve to improve our ability to understand the underlying causes and actors of these phenomena (Andrienko \& Andrienko, 1999; Jeansoulin, 2016). On the one side, visualization parameters (e.g. visual variables; Bertin, 1967) and the quality of the graphic displays play an important role in terms of the interpretation of the visual scene and discovering less salient information. On the other side, it is crucial to choose the right methods to guide efficient map use and to define the level of human-machine interaction leveraging the research on human visuospatial cognition. In this context, we need a systematic understanding of human factors affecting the spatial cognition to be able to provide personalized displays where human and machine work as a team, particularly, to create interfaces adapting to the user behavior and not the other way around. As Basole (2019) explained, instead of dictating what is shown and how, future AI-based visualization systems can learn from user behavior and recommend further actions based on users' choices and preferences.

To improve the performance of map users and to contribute to the comprehension of unknown spatial datasets, our goal is to create new ways of interactive geovisualizations by applying artificial intelligence methods. This visualization system will ideally be designed as a map interface for large touch screens involving efficiently controlled visual examination tools for reviewing and understanding unknown spatial datasets. To the best of our knowledge, machine learning algorithms (e.g. Convolutional Neural Networks, Self-Organizing Maps) are mostly developed for raster data such as remotely sensed (e.g. Ding et al., 2018), LIDAR (e.g. Kumar et al., 2019) or health and demographic survey data (e.g. Koua \& Kraak, 2004). However, we aim to explore the possibilities to apply these algorithms to vector spatial data (e.g. Yan et al., 2019) and accordingly to define the key features and functionalities of the suitable AI tools and visualization parameters. A considered vector dataset for our use case is a vector spaghetti situation from the topographic database of the National Land Survey of Finland (NLS) that can also serve as a training dataset (source: vector tile service of the NLS).

Nevertheless, it is important to include both system designers/cartographers and the focus group of (expert) users within the designing/prototyping stage, especially to learn about the user preferences, abilities and limitations. Once the experimental map interface is developed, the functionality of it and the influence of the visual variables and map features used in it are planned to be tested in real or natural operating situations using mixed methods of eye tracking and structured surveys. Touch screens in which the designed map interface will be displayed allow interactions such as zooming, selecting and panning. Additionally, in the future, we aim at integrating eye tracking not only for usability testing but also as a tool for an alternative user interaction to manipulate the map design. In other words, we intend to create a system that can adapt to the users' eye movement behavior and enable controlling the geovisualization through eye movements and, therefore, assist the information extraction on regions where the eyes are looking at (see Figure 1).
\end{abstract}




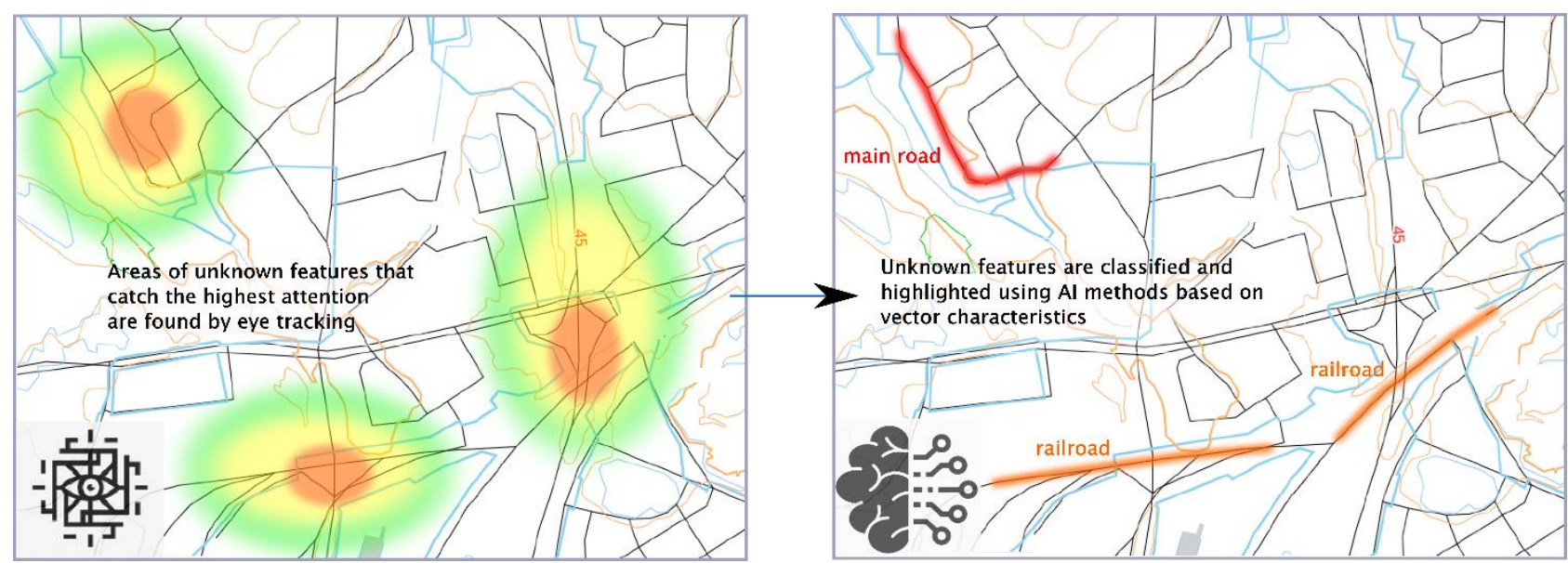

Figure 1. Exemplary picture for the AI-assisted interactive map interface controlled by eye movements.

\section{Acknowledgement:}

This research is funded by the Finnish Scientific Advisory Board for Defence (MATINE) through the TUGEVA project. 